\title{
AEBP1 as a potential immune-related prognostic biomarker in glioblastoma: a bioinformatic analyses
}

\author{
Mingjian Liu ${ }^{1,2 \#}$, Yuyu Yu ${ }^{3 \#}$, Ziqian Zhang ${ }^{1 \#}$, Zhenghong Chen ${ }^{1}$, Bin Chen ${ }^{4}$, Yijun Cheng $^{1}$, Yongxu Wei ${ }^{1}$, \\ Jia $\mathrm{Li}^{5}$, Hanbing Shang ${ }^{1}$
}

${ }^{1}$ Department of Neurosurgery, Ruijin Hospital, Shanghai Jiaotong University School of Medicine, Shanghai, China; ${ }^{2}$ Department of Neurosurgery, Shanghai East Hospital, Tongji University School of Medicine, Shanghai, China; ${ }^{3}$ Department of Oncology, RuiJin Hospital Lu Wan Branch, Shanghai Jiaotong University School of Medicine, Shanghai, China; ${ }^{4}$ Department of Neurosurgery, Yangpu Hospital, Tongji University School of Medicine, Shanghai, China; ${ }^{5}$ Institute of Neuroscience, Center for Excellence in Brain Science and Intelligence Technology, Chinese Academy of Sciences, Shanghai, China

Contributions: (I) Conception and design: H Shang, J Li, Y Cheng; (II) Administrative support: None; (III) Provision of study materials or patients: None; (IV) Collection and assembly of data: Y Yu, Z Chen, B Chen, Y Wei, Z Zhang; (V) Data analysis and interpretation: M Liu, Y Yu; (VI) Manuscript writing: All authors; (VII) Final approval of manuscript: All authors.

\#These authors contributed equally to this work.

Correspondence to: Hanbing Shang. Department of Neurosurgery, Ruijin Hospital, Shanghai Jiao Tong University School of Medicine, Shanghai 200025, China. Email: neuro_paper@126.com; Jia Li. Institute of Neuroscience, Center for Excellence in Brain Science and Intelligence Technology, Chinese Academy of Sciences, Shanghai, China. Email: lijia@ion.ac.cn.

Background: Adipocyte enhancer binding protein 1 (AEBP1) has been shown to be closely related to cancer progression; however research on its potential role in glioblastoma (GBM) remains limited.

Methods: Following an expression analysis of AEBP1 in GBM through the Oncomine database, other critical findings were accessed via The Cancer Genome Atlas (TCGA) and Genotype Tissue Expression (GTEx) databases. Specifically, in addition to identifying differentially expressed genes, the Gene Ontology and Kyoto Encyclopedia of Genes and Genomes (KEGG) were further investigated. Additionally, a gene set enrichment analysis (GSEA) was performed to examine the enrichment pathways in the AEBP1 highexpression group. To examine the prognostic role of AEBP1 in GBM, survival information was obtained from the Chinese Glioma Genome Atlas (CGGA) database. Finally, the relationship between the expression of AEBP1 and immune infiltration in GBM was examined by using the "Gene Module", "Survival Module", and "SCNA Module" on the website "Tumor Immune Estimation Resource (TIMER)".

Results: The Oncomine database revealed that AEBP1 was highly expressed in GBM. The prognostic analyses of 4 independent databases (i.e., TCGA, GTEx, Oncomine, and CGGA) revealed that AEBP1 was an independent predictable marker of GBM. The results of the GSEA showed that protein export, prion disease, cytokine receptor interaction, hematopoietic cell lineage, cell adhesion molecules, apoptosis, and the complement and coagulation cascades were differentially enriched in highly expressed AEBP1 phenotypes. Hence the conclusion is that the high expression of AEBP1 is closely correlated to poor prognosis of GBM. The immune analysis demonstrated that AEBP1 copy number alteration might affect immune infiltration in GBM tissues, and thus the survival outcomes of GBM patients.

Conclusions: High AEBP1 expression in GBM is closely correlated to patient prognosis. AEBP1 is a potential therapeutic target for the inhibition of cancerous progression and the development of new immunotherapies for GBM.

Keywords: Glioblastoma (GBM); adipocyte enhancer binding protein 1 (AEBP1); prognosis; The Cancer Genome Atlas (TCGA); the Chinese Glioma Genome Atlas (CGGA); immune infiltration

Submitted Aug 26, 2021. Accepted for publication Nov 10, 2021.

doi: $10.21037 / \mathrm{atm}-21-5183$

View this article at: https://dx.doi.org/10.21037/atm-21-5183 


\section{Introduction}

Glioma is the most common primary tumor arising from the central nervous system (CNS) in adults, and glioblastoma (GBM) is one of the most aggressive types (1). Due to its high recurrence rate, the prognosis of GBM is rather poor $(2,3)$. Further, despite decades of efforts by surgeons and researchers, there are still challenges in its treatment. More in-depth investigations on the mechanism of GBM initiation and progression are required for individualized treatment.

The identification of new effective biomarkers is a critical process, as such biomarkers not only predict patient prognosis, but could also potentially become new therapeutic targets for GBM (4-7). Adipocyte enhancer binding protein 1 (AEBP1) is a ubiquitously expressed protein, which was originally reported as a transcriptional repressor of the adipose $\mathrm{P} 2$ gene in preadipocytes (8). Additionally, AEBP1 is involved in the regulation of many tumors, such as ovarian and bladder malignancies $(9,10)$. Similarly, AEBP1 also affects the occurrence and development of GBM (11). Relevant reports have shown that the high expression of AEBP1 has positive effects on the growth, survival, and apoptosis of GBM inhibition cells $(12,13)$. We systematically studied the relationship between AEBP1 and GBM prognosis, and verified it with resources from different databases. And the unique and complex immune microenvironment of glioma is an important obstacle to immunotherapy (14). Therefore, we will study the potential mechanism and influence of AEBP1 and glioma immune microenvironment, which may help improve the efficacy of immunotherapy.

In the current study, we first examined the prognostic role of AEBP1 in GBM using multiple bioinformatic methods. A number of available databases, including Oncomine, Gene Expression Profiling Interactive Analysis (GEPIA), The Cancer Genome Atlas (TCGA), Genotype-Tissue Expression (GTEx), and Chinese Glioma Genome Atlas (CGGA), were integrated to investigate the comprehensive effects of AEBP1 on GBM. Figure 1 shows this study's workflow.

We present the following article in accordance with the REMARK reporting checklist (available at https://dx.doi. org/10.21037/atm-21-5183).

\section{Methods}

\section{AEBP1 analysis in the Oncomine database}

Oncomine (http://www.oncomine.org) integrates the transcriptomes of many major types of cancer and their respective normal tissues, and is a cancer microarray database and web-based data mining platform. It contains 715 gene expression data sets from 86,733 cancer and normal tissues (15). In this study, we used 5 glioma studies to examine the expression of AEBP1, including the Liang brain analysis, the Lee brain analysis, Bredel Brain 2 analysis, the Sun brain analysis, and the Rickman brain analysis from the Oncomine database (16-20). A P<0.01, FC of 2, and Gene Rank < top $10 \%$ were considered significant.

\section{AEBP1 analysis in the GEPIA database}

The GEPIA (http://gepia.cancer-pku.cn/) database was used to study the correlation between AEBP1 and GBM. GEPIA integrates 8,587 normal samples and 9,736 tumor samples from TCGA and GTEx projects to analyze ribonucleic acid (RNA) sequencing data, which were developed by Peking University (21). We performed a differential expression analysis of AEBP1 using a tumor and normal tissues, and conducted a survival analysis of patients in the GEPIA database.

\section{TCGA and GTEx data analysis}

To examine messenger RNA (mRNA) expression in normal brain tissues, we downloaded GTEx data $(1,152$ normal brain tissues) from (22) the UCSC Xena (http:// xena.ucsc.edu/) and used a Perl (23) script to convert the Ensembl number of the gene to the Official Symbol of the gene for the subsequent analysis. Next, the R packages of gganatogram (24) and ggpubr on the GitHub (25) were used to examine the expression of AEBP1 in various organs of the human body. Combined with the GBM data downloaded from TCGA database (5 paracancer tissues and $160 \mathrm{GBM}$ samples) (http://cancergenome.nih.gov/), we used (26) the limma package to screen DEGs $(\mid \operatorname{logFCI}>2$, FDR $<0.05)$ and used the (27) heatmap package to plot a heatmap. The clusterProfiler package was then used for the GO and KEGG pathway analyses (28), and the GOplot package was used for the cluster analysis (29). We used the GSEA program to investigate the functions correlated with high and low AEBP1 expression levels in the TCGA cohort (30).

\section{CGGA data analysis}

The data of 389 GBM patients were downloaded from the CGGA (www.cgga.org.cn) database. Due to incomplete information, 100 patients were excluded from the study, 


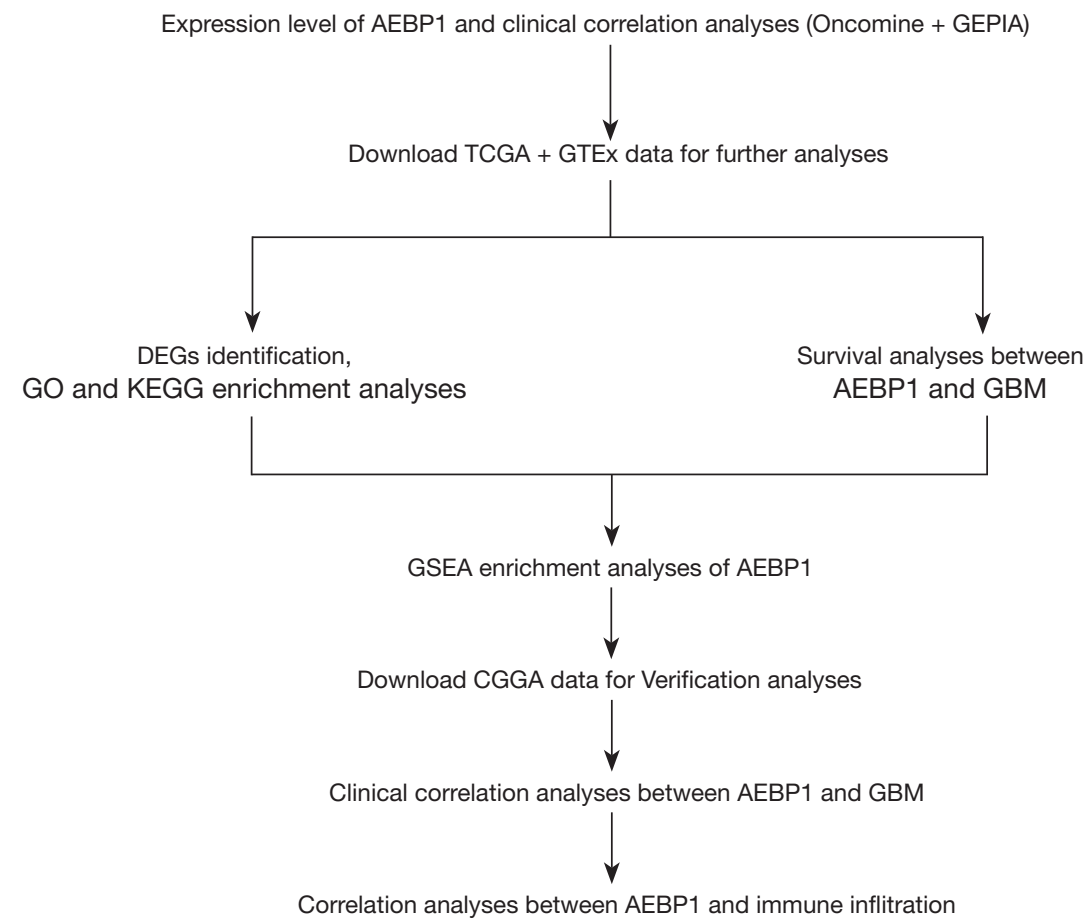

Figure 1 Study flow chart.

and we only analyzed the data of the remaining 289 patients. Kaplan-Meier curves were plotted to show the relationship between the OS of patients and the expression level of AEBP1. We then used beeswarm plots to show the relationship between different clinical features and AEBP1 expression.

\section{AEBP1 analysis in the TIMER database}

TIMER (https://cistrome.shinyapps.io/timer/) is a website that comprehensively analyzes the immune invasion of different tumors (31). The website uses statistical methods to estimate the abundance of 6 immune cells (B cells, CD8 ${ }^{+}$ $\mathrm{T}$ cells, macrophages, neutrophils, $\mathrm{CD}^{+} \mathrm{T}$ cells, and dendritic cells) in GBM. We then used the "Gene Module", "Survival Module", and "SCNA Module" on the website to examine the correlation between immune infiltration and the expression of AEBP1 in GBM patients.

The study was conducted in accordance with the Declaration of Helsinki (as revised in 2013).

\section{Statistical analysis}

All data were shown as mean \pm standard error of the mean
(SEM). Multiple different groups were analyzed with a oneway analysis of variance (ANOVA) followed by Bonferroni test. A P value $<0.05$ was considered statistically significant.

\section{Results}

\section{AEBP1 expression in GBM tissues and normal buman brain tissues}

First, we used the Oncomine database to detect the expression pattern of AEBP1 in GBM tissues. After screening, a total of 5 studies showed that AEBP1 was upregulated in CNS cancers, but its downregulation tendency was not reported (see Figure $2 A, 2 B$ ). In each study, the expression level of AEBP1 in glioma tissues was significantly higher than that in control peritumoral tissues (see Figure 3A-3E). Among the 950 different AEBP1 outlier analysis studies, 7 studies showed that the expression of AEBP1 was upregulated, and 6 studies showed that it was downregulated. To further determine whether AEBP1 was associated with glioma malignancies, a cancer versus cancer analysis (see Figure $4 A$ ) was conducted that showed that in 5 of the 6 studies, AEBP1 was significantly more highly expressed in GBM than in other glioma counterparts 
A

Disease summary for AEBP1

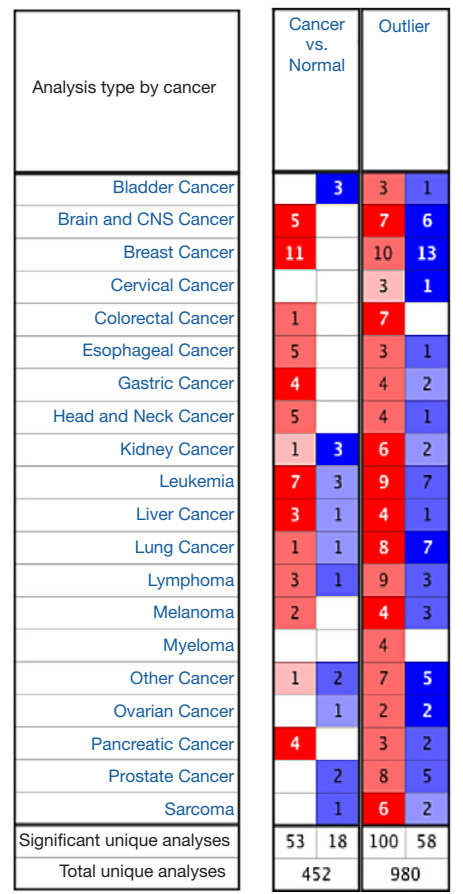

B

Comparison of AEBP1 across 5 analyses

Over-expression

\begin{tabular}{|c|c|c|c|c|c|c|c|}
\hline Median rank & $\mathrm{P}$-value & Gene & & & & & \\
\hline \multirow[t]{2}{*}{342.0} & $2.82 \mathrm{E}-7$ & AEBP1 & & & & & \\
\hline & & & 1 & 2 & 3 & 4 & 5 \\
\hline
\end{tabular}

Legend

1. Glioblastoma vs. Normal Bredel 4. Astrocytoma vs. Normal Rickman

Brain 2, Cancer Res, $2005 \quad$ Brain, Cancer Res, 2001

2. Glioblastoma vs. Normal

Lee Brain, Cancer Cell, 2006

5. Glioblastoma vs. Normal Sun

Brain, Cancer Cell, 2006

3. Glioblastoma vs. Normal Liang Brain,

Proc Natl Acad Sci USA, 2005

$151025 \quad 25105 \quad 1$

$\square \square \square \square \square \square \square \square \square \square$ Not measured

The rank for a gene is the median rank for that gene across each of the analyse. The P-value for a gene is its $\mathrm{P}$-value for the median-ranked analysis.

Figure 2 AEBP1 expression in glioma tissues. (A) Disease summary for AEBP1. (B) Comparison of AEBP1 over-expression in 5 analyses. $\mathrm{P}<0.05$. AEBP1, adipocyte enhancer binding protein 1 .

(see Figure 4B-4G). These results indicate that AEBP1 is differently expressed in glioma and may be a potential biomarker of GBM progression.

GTEx data were then used to compare the expression level of AEBP1 in GBM and normal human brain tissues. As Figure $5 A, 5 B$ show, the lower expression of AEBP1 was only detected in seldom tissues, compared with brain tissue. No differences between males and females were observed (see Figure 5C), and subsequent analyses also indicated that the data of different sexes could be unbiasedly integrated.

\section{Relationship between the prognosis of GBM and AEBP1 expression and the prognostic role of AEBP1 in GBM}

To verify the analytic results from the Oncomine database and further explore correlations between AEBP1 expression and GBM, we analyzed relevant data from the GEPIA database, and confirmed that AEBP1 was significantly upregulated in GBM, acute myeloid leukemia, head and neck squamous cell carcinoma, thymoma, skin cutaneous melanoma, lymphoid neoplasm diffuse large b-cell lymphoma, and pancreatic adenocarcinoma (see
Figure 6A). Conversely, endocervical adenocarcinoma and cervical squamous cell carcinoma, adrenocortical carcinoma, and kidney chromophore showed a decreased expression pattern of AEBP1. Interestingly, compared to normal brain tissue, the upregulation of AEBP1 was observed in highly aggressive GBM but not in low-grade glioma (see Figure 6B). A further prognostic analysis showed that the higher the expression of AEBP1, the shorter the overall survival (OS) of GBM patients (see Figure 6C). Thus, we found that AEBP1 was significantly upregulated in GBM and possessed a prognostic value for GBM. Together, these results suggest that $\mathrm{AEBP} 1$ plays a pro-cancerous role in GBM.

\section{Bioinformatic Analysis of TCGA and GTEx Database}

Through a differentially expressed gene (DEG) analysis, a total of 1,923 DEGs were identified from the GTEx and TCGA data [ $\mid$ fold change (FC) $\mid>2$, false discovery rate $(\mathrm{FDR})<0.05$ ] (see https://cdn.amegroups.cn/static/ public/atm-21-5183-1.xlsx and Figure 7). A further Gene Ontology (GO) analysis showed that these DEGs were 
A AEBP1 expression in Bredle Brain Over-expression gene rank: top $1 \%$

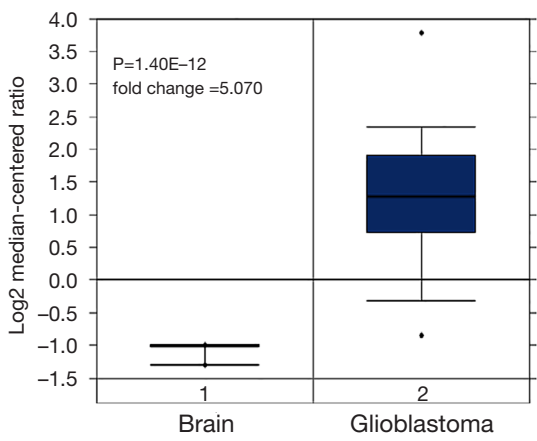

D AEBP1 expression in Rickman Brain Over-expression gene rank: top $10 \%$

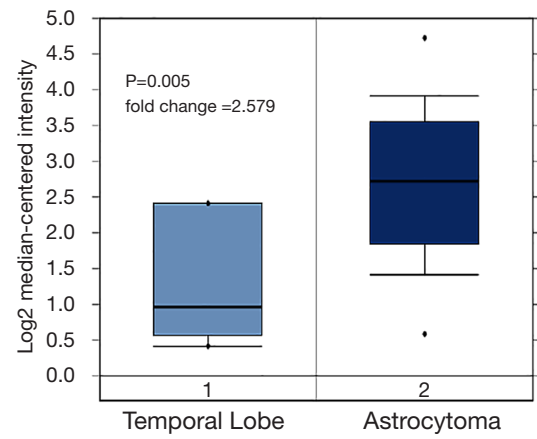

B

AEBP1 expression in Liang Brain Over-expression gene rank: top $2 \%$

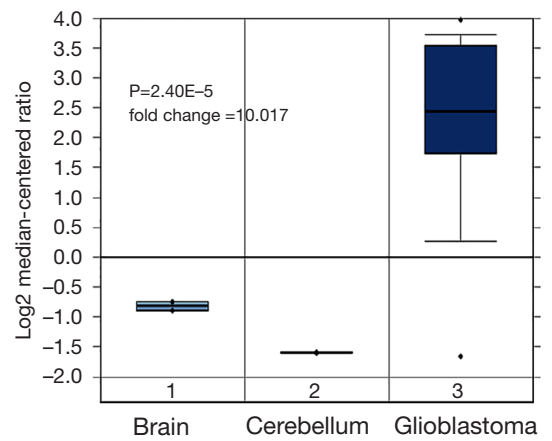

E

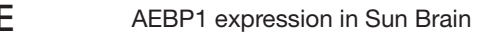
Over-expression gene rank: top $5 \%$

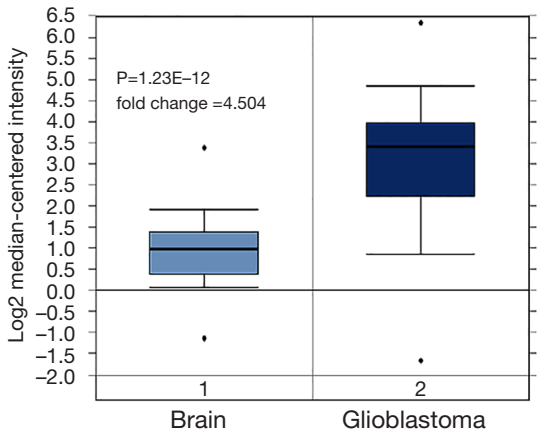

C

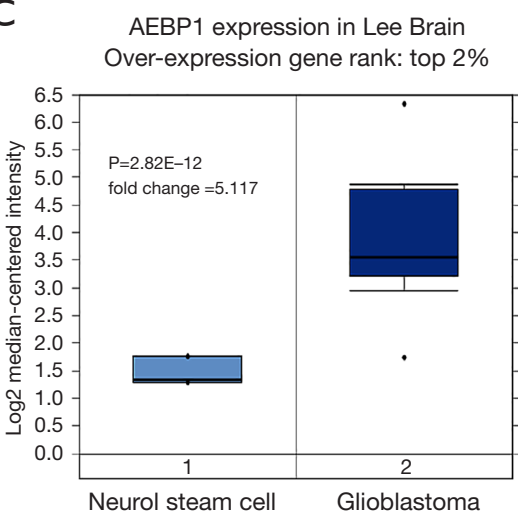

Figure 3 AEBP1 expression in cancer $v$ s. normal tissues in 5 analyses. (A) Bredel brain, (B) Liang brain, (C) Lee brain, (D) Rickman brain, and (E) Sun brain. $\mathrm{P}<0.01$. AEBP1, adipocyte enhancer binding protein 1 .

significantly enriched in the regulation of vesicle-mediated transport, neutrophil degranulation, and the modulation of chemical synaptic transmission in the biological processes. The cellular components were mainly enriched in the presynapse, extracellular matrix, and neuronal cell body. The molecular functions were significantly enriched in phospholipid binding, substrate-specific channel activity, and ion channel activity (see Figure $8 A$ ). The Kyoto Encyclopedia of Genes and Genomes (KEGG) pathway analysis of the DEG showed that it was mainly concentrated in cell adhesion molecules and immune-related signaling pathways (see Figure 8B). We then performed cluster analyses of the top pathways and GO terms. The top GO terms were enriched in neurotransmitter secretion, neurotransmitter transport, the regulation of vesiclemediated transport, the signal release from synapse, and the modulation of chemical synaptic transmission (see Figure $8 C$ ). The pathways were enriched in the phagosome, type I diabetes mellitus, viral myocarditis, GABAergic synapse, and Epstein-Barr virus infection (see Figure $8 D$ ). Further, similar to the results of the GEPIA analysis, the survival curve of AEBP1 (see Figure 8E) showed that GBM patients with low AEBP1 expression lived longer than those with high AEBP1 expression (the $\mathrm{P}$ value was much less than 0.001 ).

\section{Gene sets enriched in AEBP1 expression phenotype}

To further examine the biological role of AEBP1 in GBM, a GSEA was performed (see Figure 9). The results showed that a series of functions, including apoptosis, antigen processing and presentation, the complement and the coagulation cascades, the B-cell receptor signaling pathway, lysosome, pathways in cancer, the toll-like receptor signaling pathway, and the $\mathrm{T}$-cell receptor signaling pathway, were differentially enriched in the AEBP1 high- 
A

Comparison of AEBP1 across 6 analyses

Over-expression

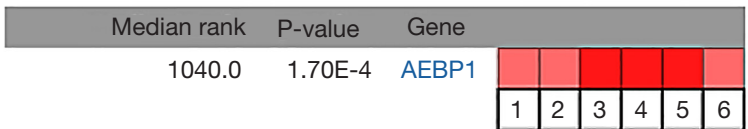

Legend

1. Brain and CNS Cancer type : Glioblastoma

4. Brain and CNS Cancer Type: Glioblastoma

Bredel Brain 2, Cancer Res, 2005

Liang Brain, Proc Natl Acad Sci USA, 2005

2. Brain and CNS cancer type: Glioblastoma

5. Brain and CNS cancer type: Glioblastoma

Freije Brain, Cancer Res, 2004

Nutt Brain, Cancer Res, 2003

3. Neuroblastic tumor type: Ganglioneuroma

6. Brain and CNS Cancer Type: Glioblastoma

Janoueix-Lerosey Brain, Nature, 2008

Sun Brain, Cancer Cell, 2006

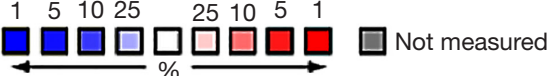

The rank for a gene is the median rank for that gene across each of the analyse.

The P-value for a gene is its P-value for the median-ranked analysis.

B

AEBP1 expression in Liang Brain Over-expression gene rank: top $4 \%$

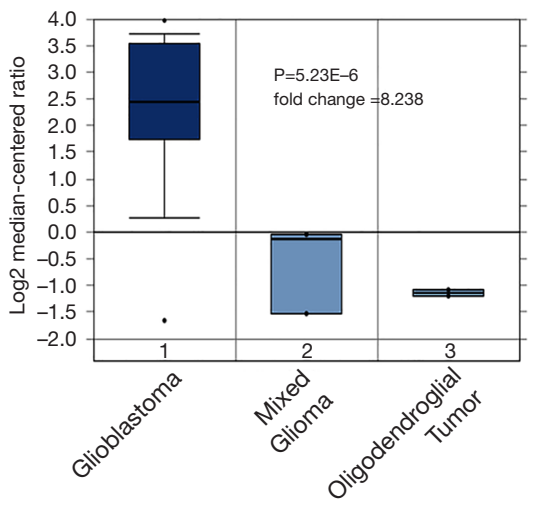

$E$

AEBP1 expression in Bredel Brain 2 Over-expression gene rank: top $8 \%$
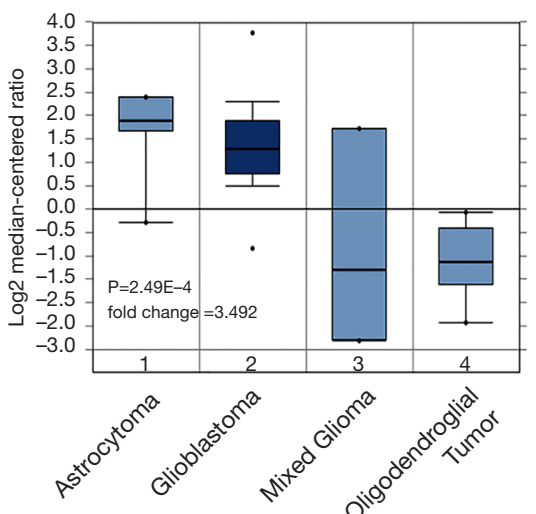

C

AEBP1 expression in Nutt Brain

Over-expression gene rank: top $4 \%$

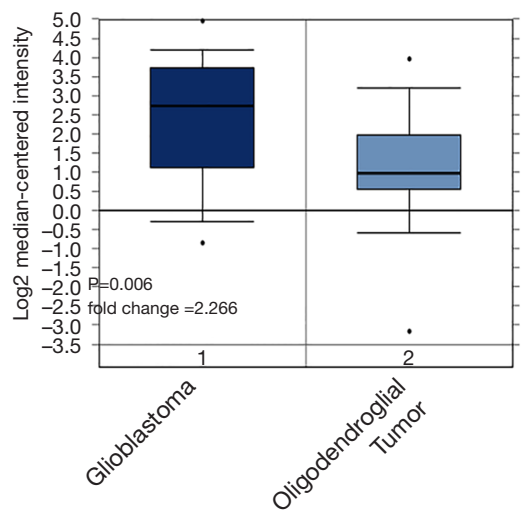

$\mathrm{F}$
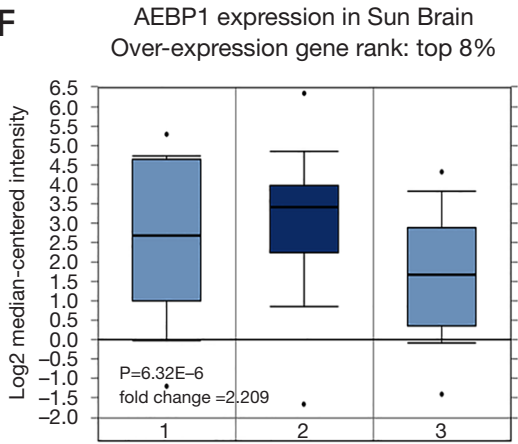

Over-expression gene rank: top 8\%

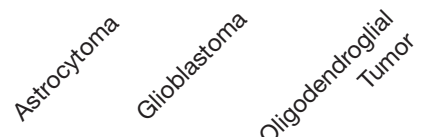

D AEBP1 expression in Janoueix-Lerosey Brain Over-expression gene rank: top $5 \%$

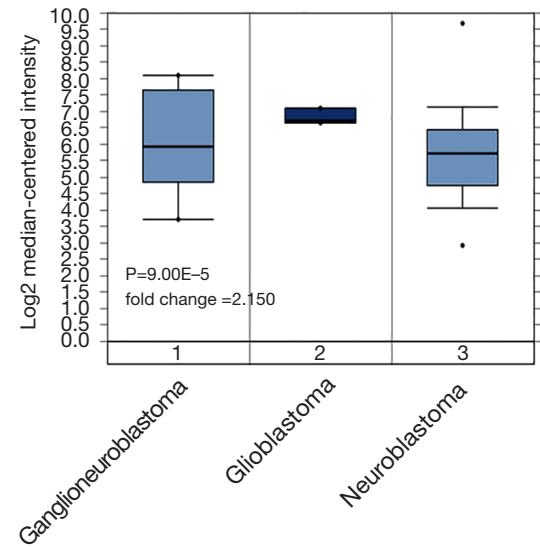

G

AEBP1 expression in Freije Brain

Over-expression gene rank: top $10 \%$
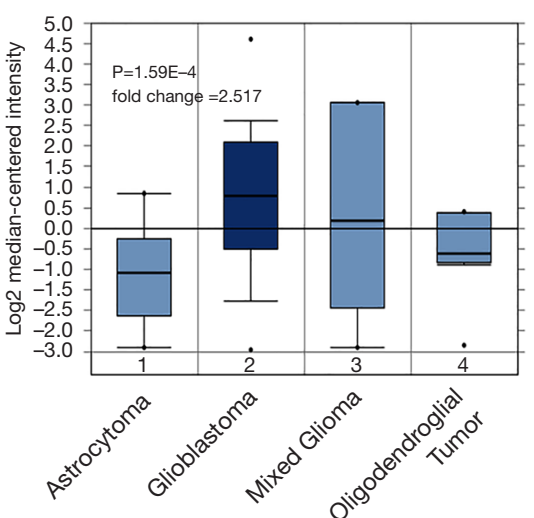

Figure 4 AEBP1 expression in cancer $v$ s. cancer tissues analysis. (A) Comparison of AEBP1 expression across 6 analyses. AEBP1 expression in (B) Liang brain, (C) Nutt brain, (D) Janoueix-Lerosey brain, (E) Bredel brain 2, (F) Sun brain and (G) Freije brain. P<0.01. AEBP1, adipocyte enhancer binding protein 1 . 
A

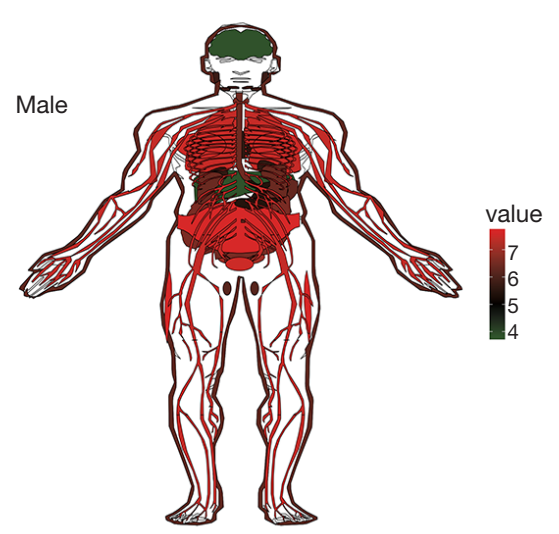

B

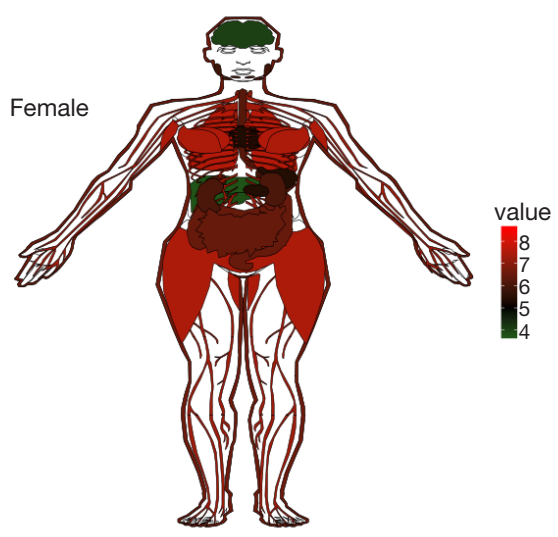

C Gender 官 Female 官 Male

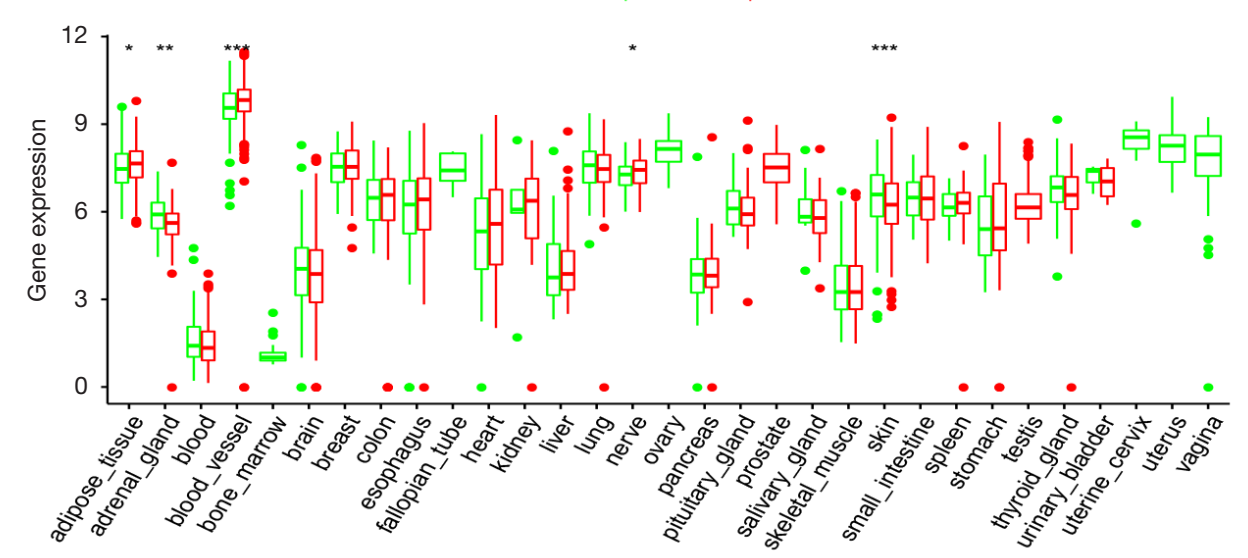

Figure 5 AEBP1 expression in normal human tissues. (A) The expression level of AEBP1 in various organs in (A) men and (B) women (red represents high expression, green represents low expression). (C) Expression level of AEBP1 in tissues from patients of different genders. *, $\mathrm{P}<0.05$; **, $\mathrm{P}<0.01$; ***, $\mathrm{P}<0.001$. AEBP1, adipocyte enhancer binding protein 1.

expression phenotype.

\section{Validation of the clinical correlation from the CGGA}

To further validate the prognostic value of AEBP1 for GBM, we downloaded the sequencing data and clinical information of 389 patients from the CGGA database. Using the Limma and beeswarm packages, we found that AEBP1 was markedly upregulated in GBM (see Figure 10A). Due to missing data, a total of 290 eligible patients were ultimately included in the prognostic analysis. Interestingly, consistent with the GEPIA and TCGA results, GBM patients with high AEBP1 expression had worse survival outcomes (see Figure 10B). Further, the differential expression of AEBP1 was noticeably associated with age, sample type, $1 \mathrm{p} 19 \mathrm{q}$ codeletion status, and isocitrate dehydrogenase (IDH) mutation (see Figure 10C-10I).

In addition, we also examined the receiver operating characteristic (ROC) curves and undertook a logistic regression of the CGGA dataset. The ROC curves showed that risk score precisely predicted the 5-year survival rates of GBM patients [area under the curve (AUC) $=70.7 \%$ ] (see Figure 107). The univariate Cox analysis (see Figure 10K) revealed that AEBP1 [hazard ratio (HR) $=1.12$; $95 \%$ confidence interval (CI): 1.04-1.19] and sample type ( $\mathrm{HR}=1.47 ; 95 \% \mathrm{CI}: 1.232-1.758)$ were high-risk factors. Conversely, IDH mutation $(\mathrm{HR}=0.71 ; 95 \% \mathrm{CI}$ : $0.53-0.97$ ), chemotherapy (HR $=0.47 ; 95 \%$ CI: $0.34-0.65)$, and radiation therapy ( $\mathrm{HR}=0.58 ; 95 \% \mathrm{CI}: 0.41-0.82$ ) were all low-risk factors. A further multivariate analysis 

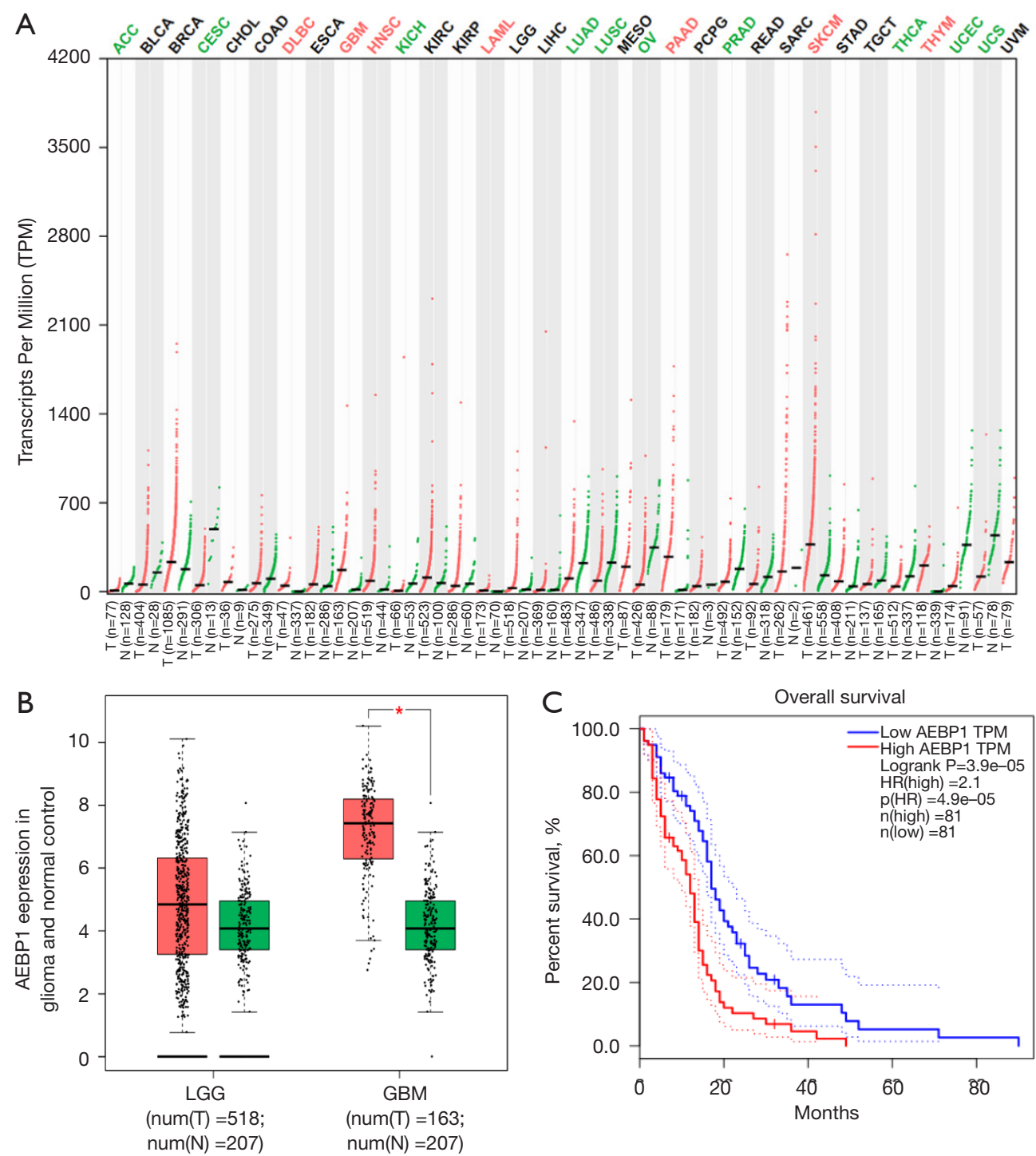

Figure 6 The relationship between the prognosis of GBM patients and AEBP1 expression. (A) AEBP1 expression was significantly upregulated in a variety of tumors. (B) The expression level of AEBP1 was significantly higher than that of normal controls in GBM. *, $\mathrm{P}<0.05$. (C) OS curves of AEBP1 in the GEPIA. GBM, glioblastoma; AEBP1, adipocyte enhancer binding protein 1; OS, overall survival.

(see Figure 10L) confirmed that AEBP1 (HR $=1.12 ; 95 \%$ CI: $1.04-1.21 ; \mathrm{P}<0.01)$ was still independently related to the OS rate. The univariate and multivariate analyses showed that AEBP1 might be an independent prognostic marker in GBM patients. Consistent with the findings of the univariate Cox analysis, sample type (HR $=1.89 ; 95 \%$ CI: $1.54-2.32 ; \mathrm{P}<0.01)$ was a high-risk factor, and IDH mutation ( $\mathrm{HR}=0.63 ; 95 \% \mathrm{CI}: 0.44-0.90 ; \mathrm{P}=0.01)$, and chemotherapy (HR $=0.46$; 95\% CI: 0.33-0.64; $\mathrm{P}<0.01$ ) were low-risk factors.

\section{AEBP1 expression is correlated with immune-infiltration level}

The GSEA analysis indicated that the genes in the AEBP1 high-expression group were enriched in immune-related pathways. Thus, the Tumor Immune Estimation Resource (TIMER) was employed to further investigate the immune infiltration of GBM. The results showed that AEBP1 was negatively correlated with cluster of differentiation $8\left(\mathrm{CD}^{+}\right)$ $\mathrm{T}$ cells and purity, but was positively correlated with $\mathrm{CD} 4^{+}$ 


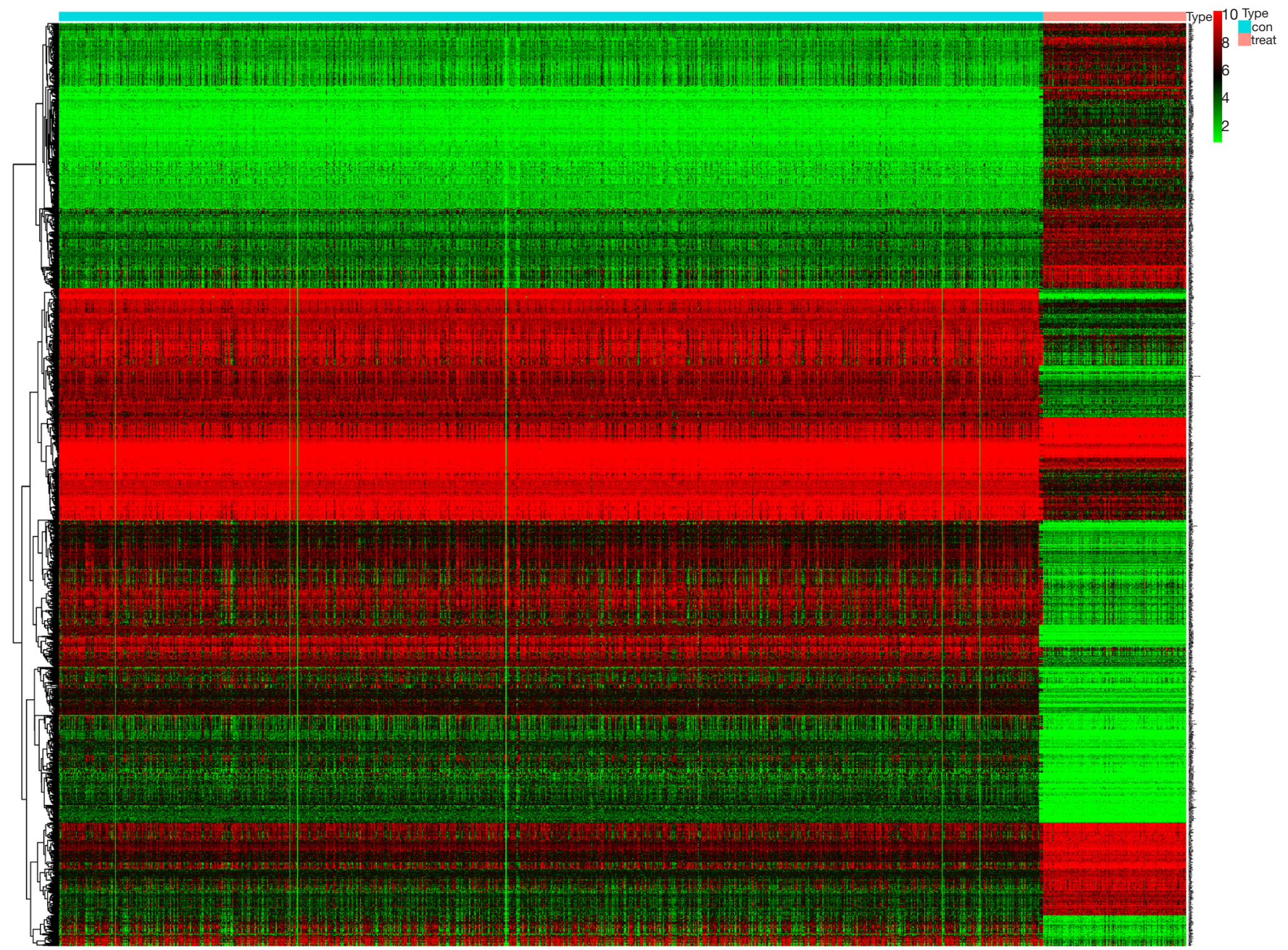

Figure 7 Heatmap of DEGs in glioblastoma. DEG, differentially expressed gene.

T cells, B cells, and dendritic cells in GBM (see Figure $11 A$ and Table 1). Next, the TIMER data were used for the univariate and multivariate Cox survival analyses. The results of the univariate analysis showed that dendritic cells and AEBP1 affected the survival outcome of GBM patients (see Figure $11 B$ and Table 2). The results of the multivariate analysis showed that age, dendritic cell count, and AEBP1 were independent prognostic factors of GBM (see Table 3). In addition, the changes in the copy number of AEBP1 appeared to significantly affect the immune-infiltration level in GBM (see Figure 11C). Together, these results highlight that the alteration of AEBP1 copy number is closely related to the immune-infiltration level and predicts the prognosis of GBM patients. Thus, AEBP1 might be a potential reference value for determining the inhibition of GBM progression and the development of new immunotherapies.

\section{Discussion}

Glioma is a common malignancy originating from the glial cells in the brain and spine (32). Due to its high invasion and recurrence rate (33-35), surgical and other interventions cannot radically cure the tumor (36). Despite continuous advancements in treatments, the prognosis of GBM remains very poor, and on average, patients survive less than 2 years (37). Thus, identifying more effective biomarkers is critical to individualized therapy and prognostic prediction.

AEBP1 has been reported to be correlated with the initiation and progression of GBM $(38,39)$; however, the mechanism underlying AEBP1 in GBM requires further study. In our study, using data from the Oncomine database, we found that AEBP1 was highly expressed in GBM tissues. Our results are consistent with previous reports and indicate 
A

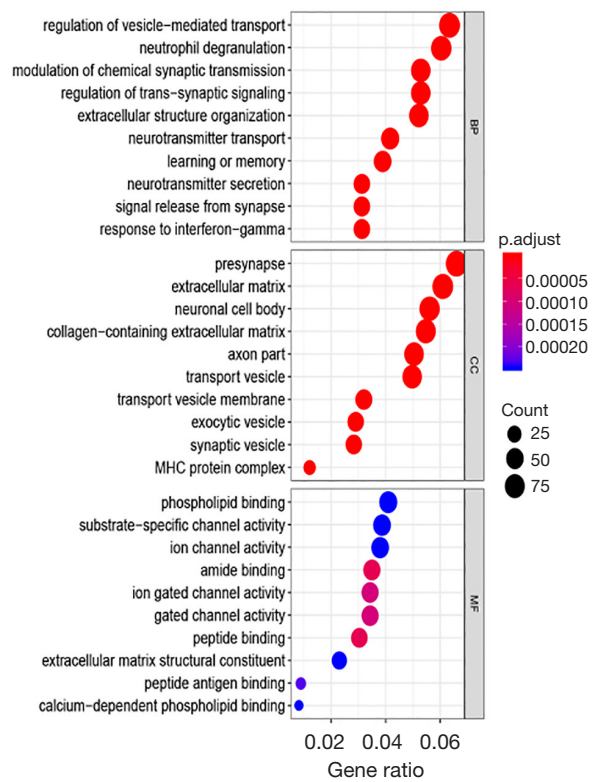

C

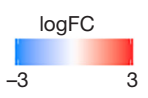

GO terms

$\square$ Requlation of vesicle-mediated

transport

Neurotransmitter secretion

Signal release from synapse

Neurotransmitter transport

Modulation of chemical synaptic transmission
B

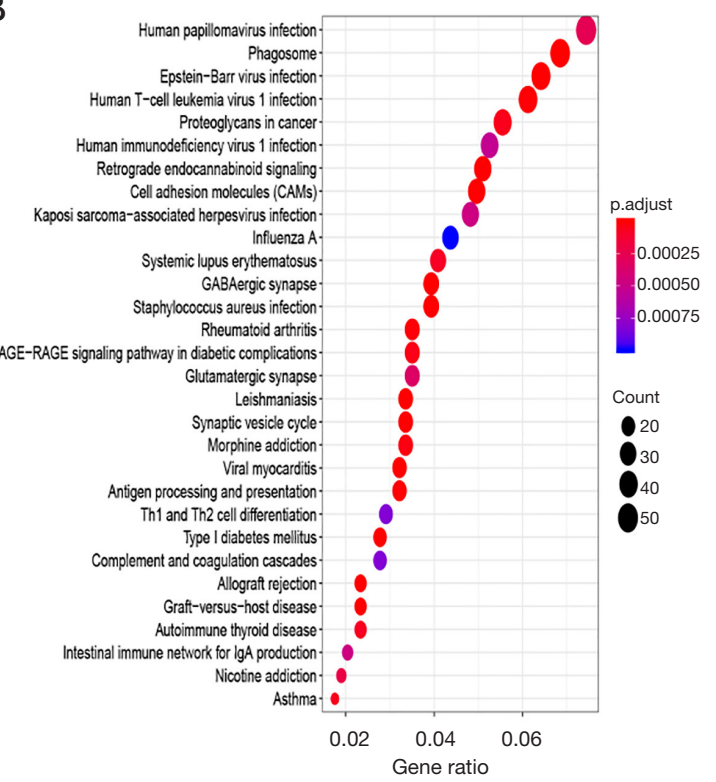

D

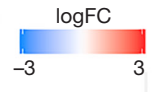

GO terms

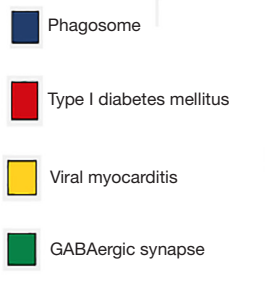

Epstein-Barr virus infection

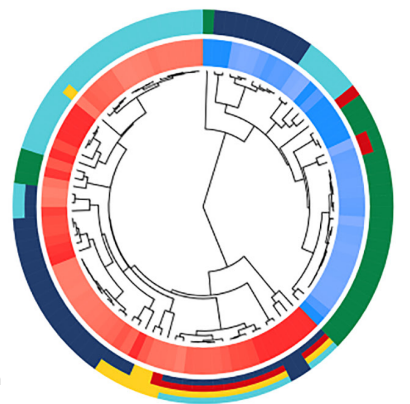

E

AEBP1 level $\rightarrow$ High $\longrightarrow$ Low
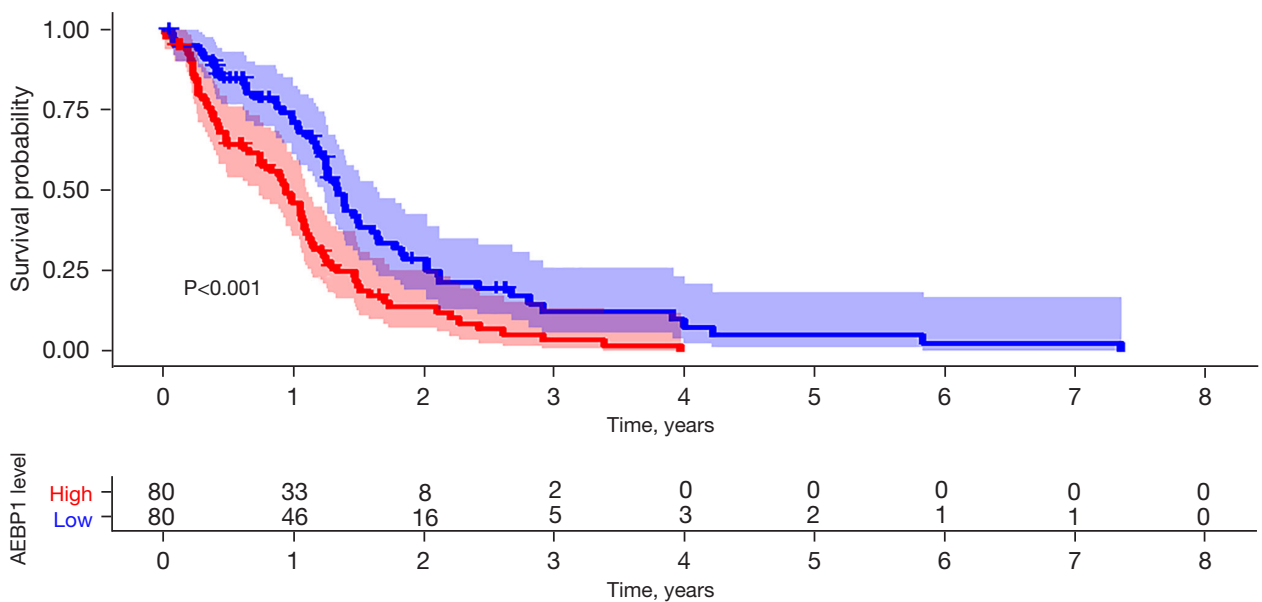

Figure 8 Bioinformatic analysis of TCGA and GTEx database. Bubble chart of GO (A) and KEGG (B) analysis of DEGs. (C) GO and (D) KEGG pathway cluster analyses. (E) Overall survival curves of AEBP1 for TCGA analysis. TCGA, The Cancer Genome Atlas; GTEx, Genotype Tissue Expression; GO, Gene Ontology; KEGG, Kyoto Encyclopedia of Genes and Genomes; DEG, differentially expressed gene; AEBP1, adipocyte enhancer binding protein 1. 

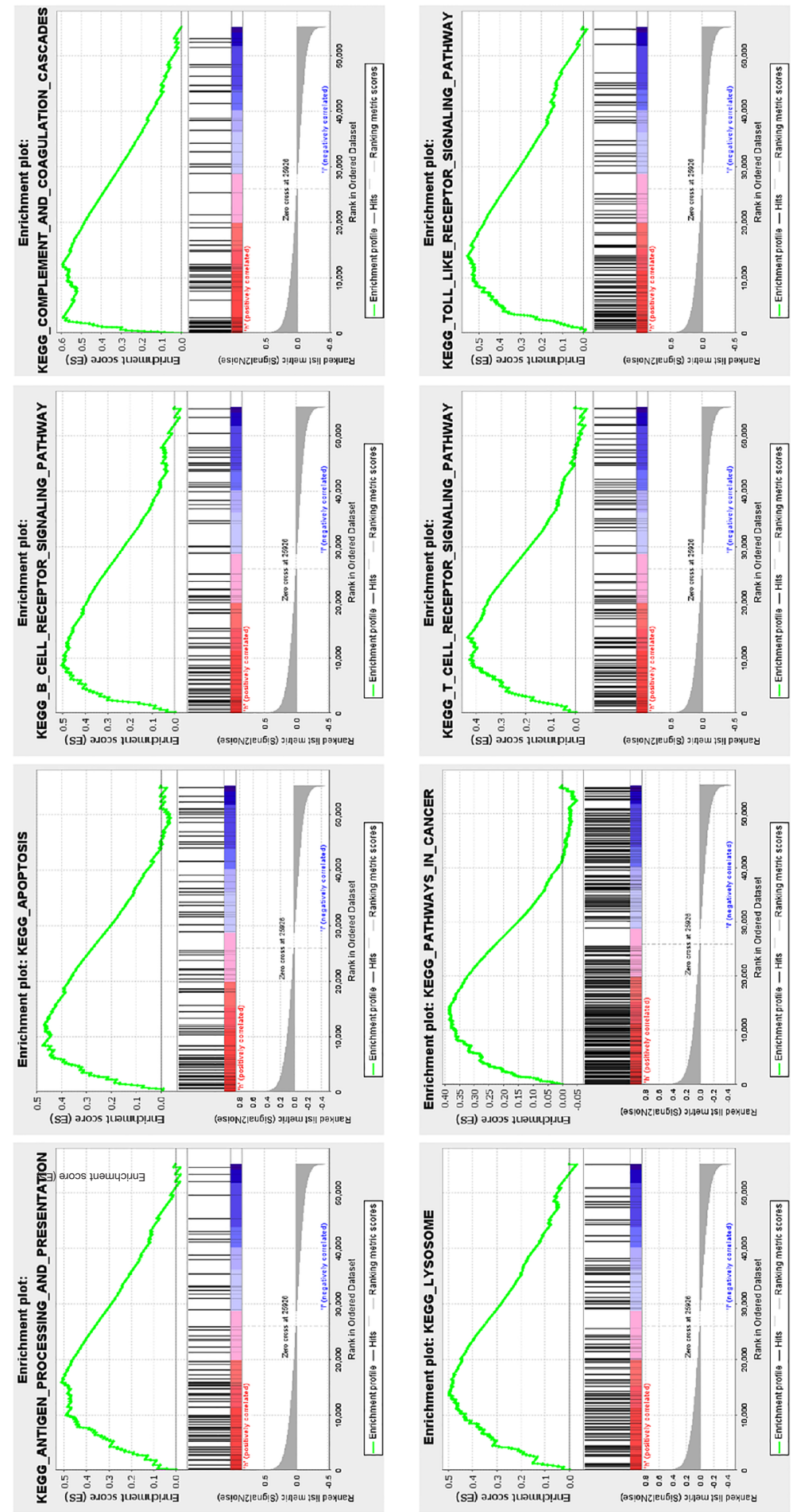

$\varangle$
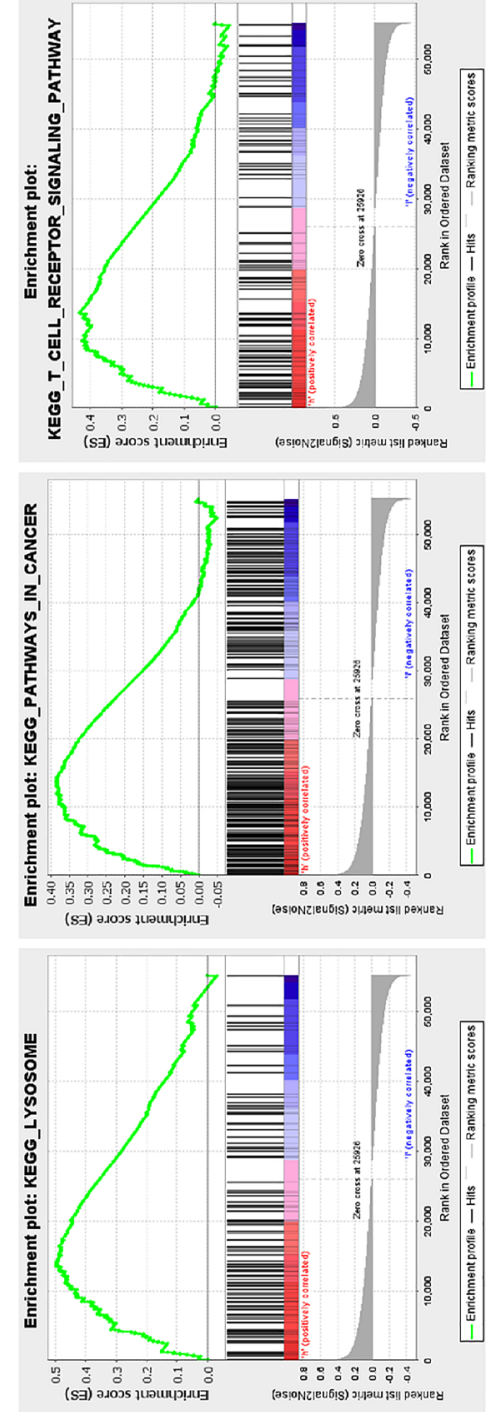

$\infty$

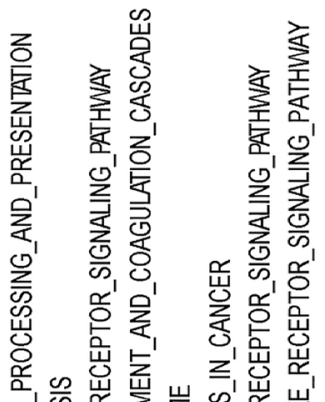

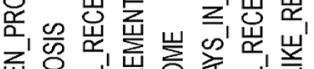

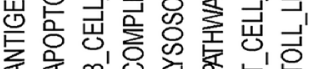
崖岀岀岀岀岀岀 | | || | || || ||

ت

苟

章

记

o

ㅊ.

$\exists$ 这

ป

远

.

츨.

ज्ञ

एक

$\because$ 政

离高

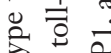

:

至

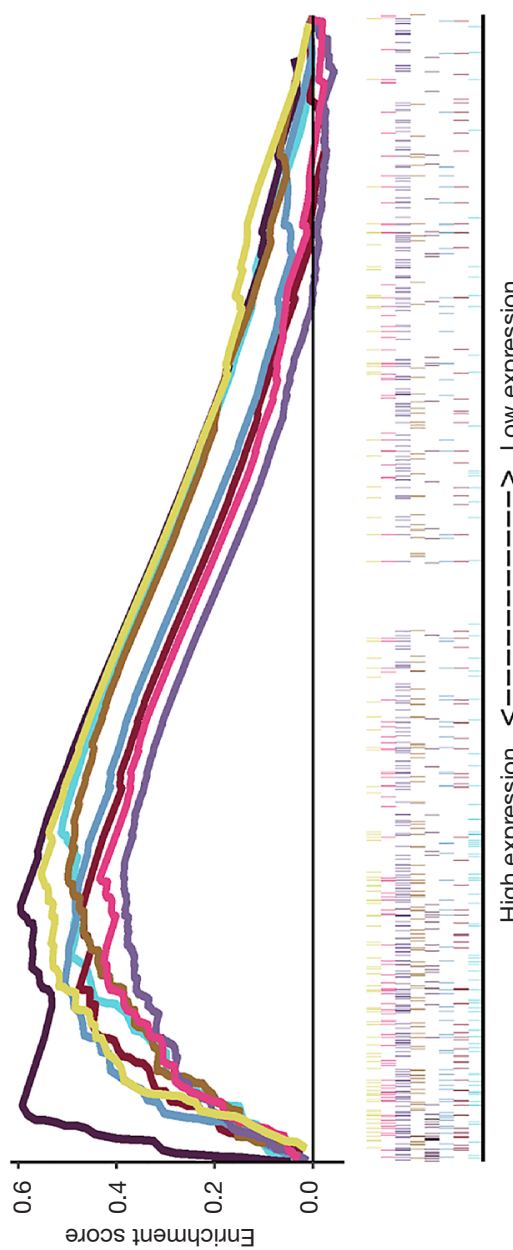

ㄱ.

$5 \stackrel{2}{2}$

位

य

焉

$\exists$.

ㅎำ

.

เे

ब

.5:

$=\frac{1}{2}$

है

\&

范

苛苛

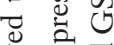

渮

के का

.

栗

匆 츨

3 .

记

जि तु

ये

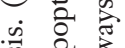

急高

जี के

出苋

0 ․

a

范 
A

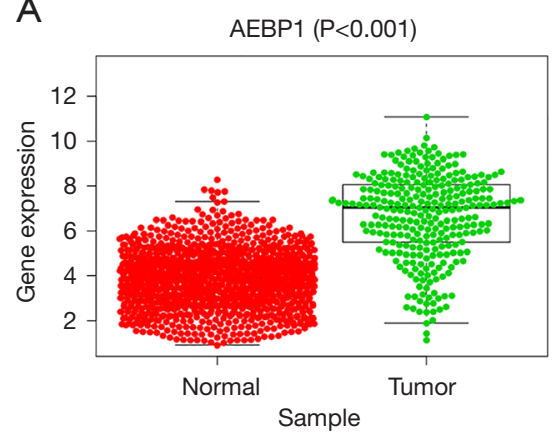

D

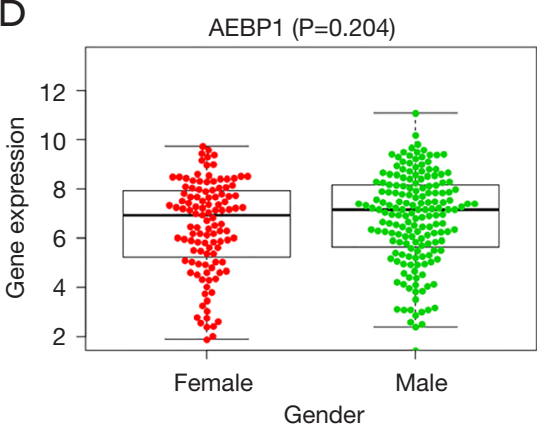

G

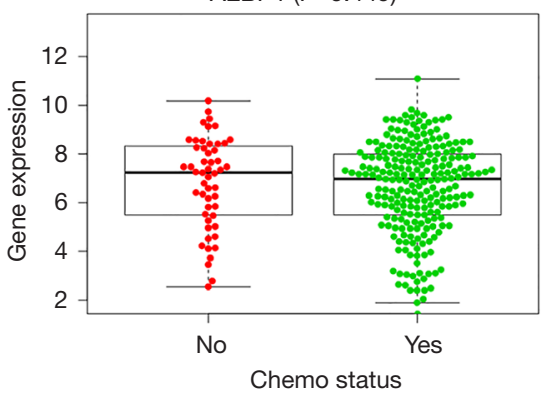

J

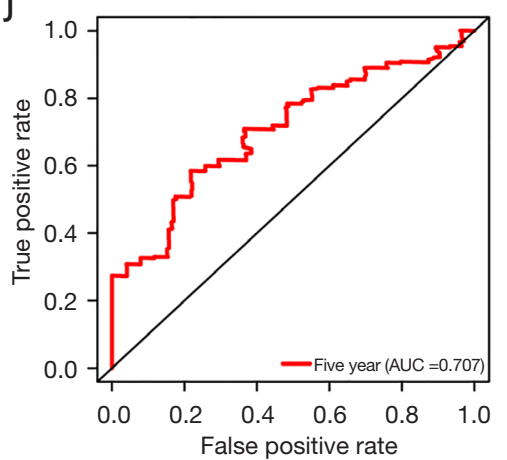

B

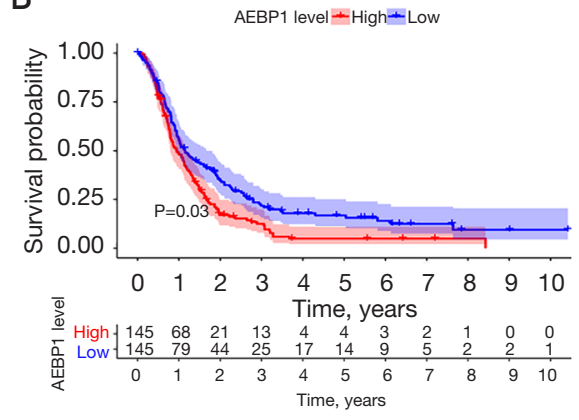

E

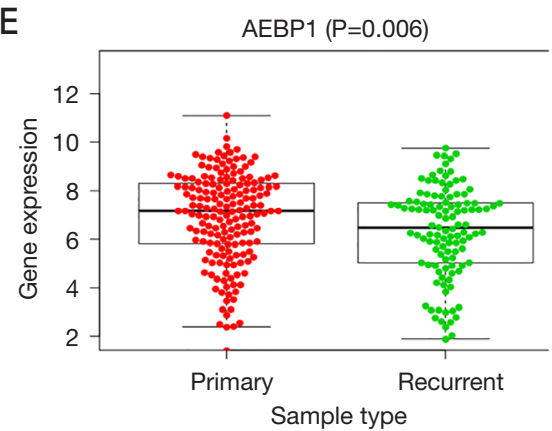

$\mathrm{H}$

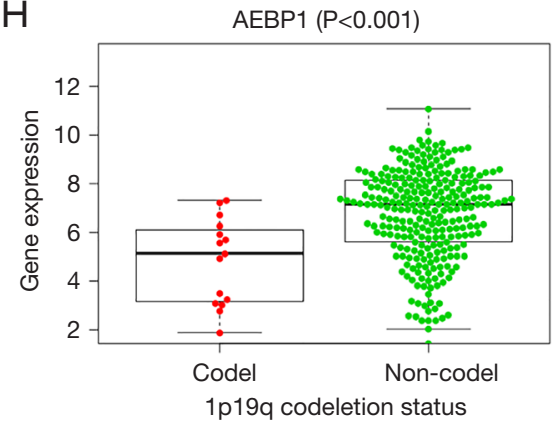

K

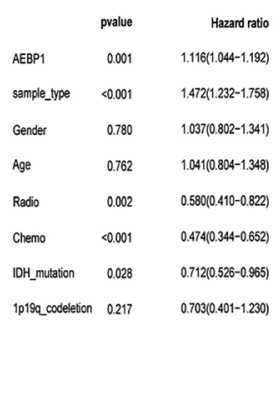

C

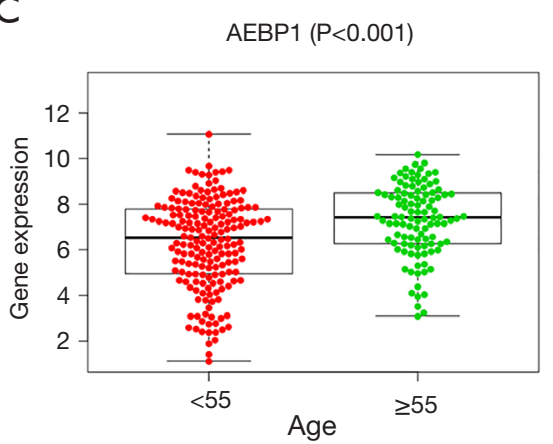

F

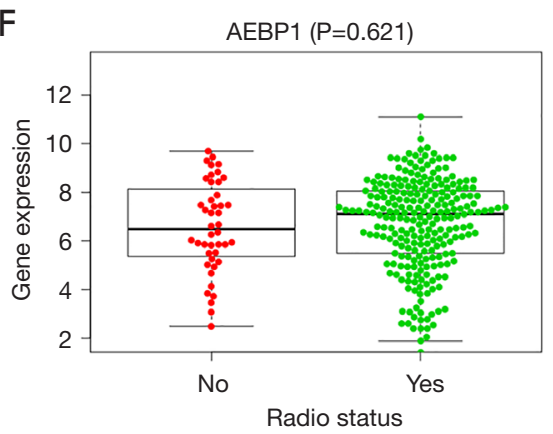

I

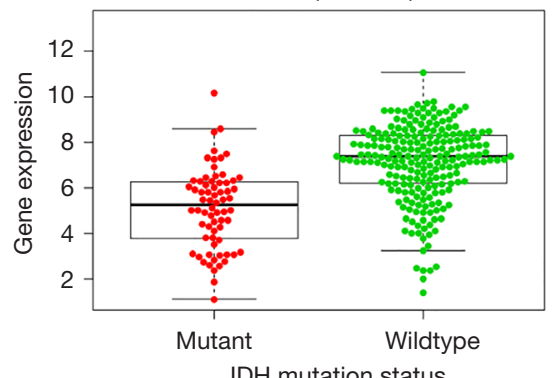

L

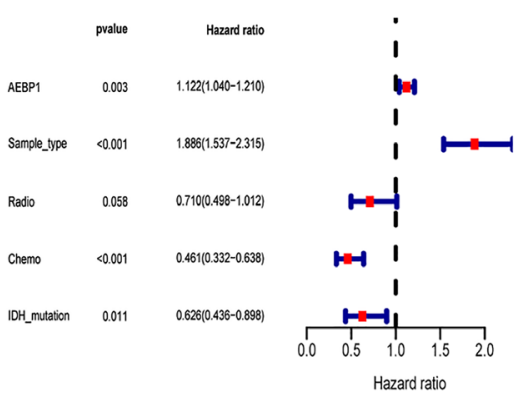

Figure 10 Correlation analysis of AEBP1 expression and clinical features. (A) Analysis of the expression level of AEBP1 in glioblastoma. (B) The relationship between the expression level of AEBP1 and the survival rate. (C-I) Analysis of the correlation between AEBP1 expression and different clinical features. (J) ROC curve showing the predictive efficiency of AEBP1. (K) Univariate and (L) multivariate Cox regression analyses of the association between the overall survival of patients and clinical factors (including AEBP1 expression). AEBP1, adipocyte enhancer binding protein 1 ; ROC, receiver operating characteristic. 

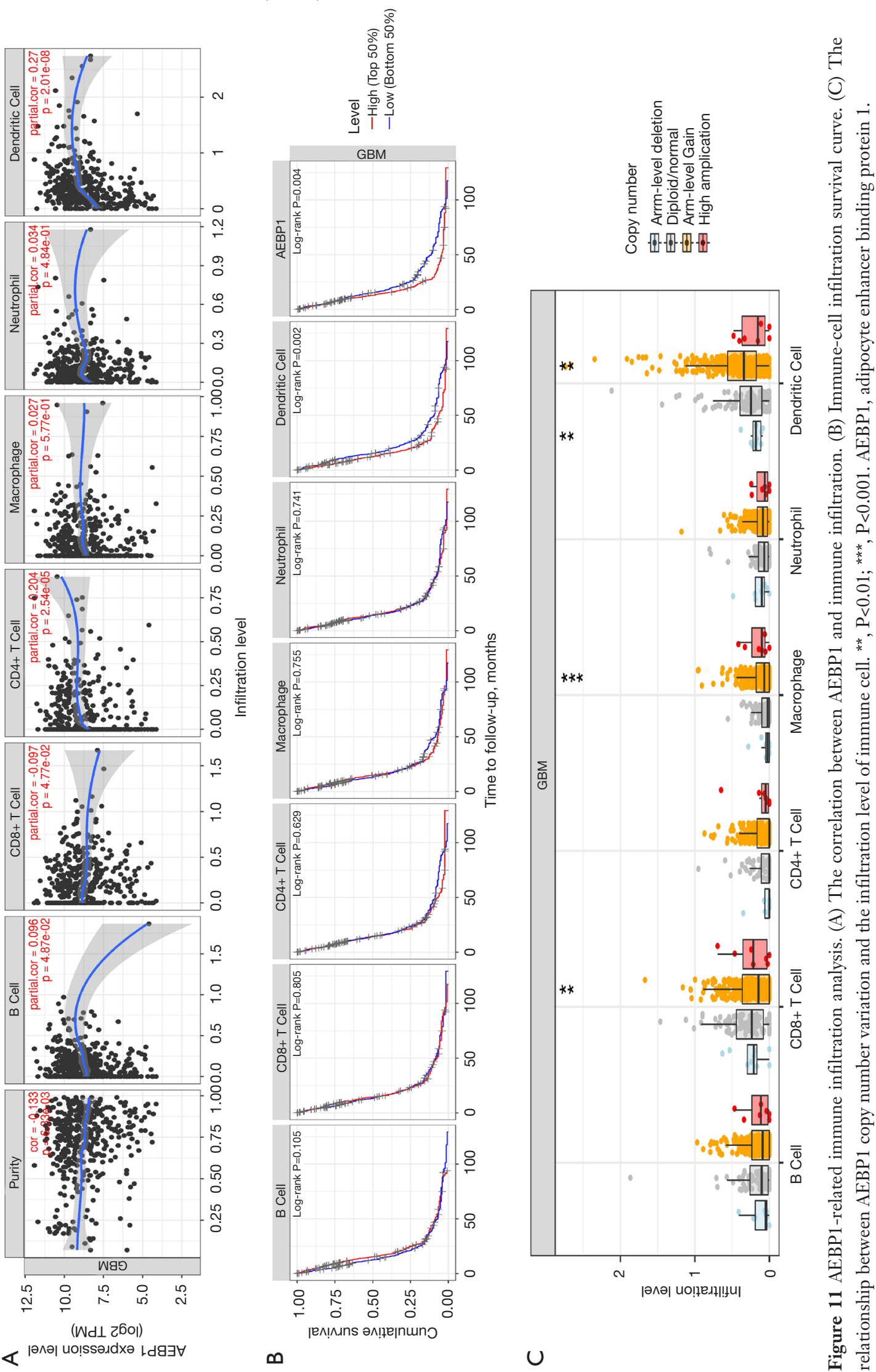

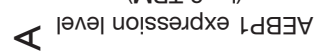


Table 1 The relationship between AEBP1 expression and immune-infiltration levels in GBM

\begin{tabular}{lccc}
\hline Cancer & Variable & Partial.cor & P \\
\hline GBM & Dendritic cell & 0.27 & 0.00 \\
GBM & CD4 $4^{+}$cell & 0.20 & 0.00 \\
GBM & Purity & 0.13 & 0.01 \\
GBM & CD8 ${ }^{+}$T cell & 0.10 & 0.05 \\
GBM & B cell & 0.10 & 0.05 \\
GBM & Neutrophil & 0.03 & 0.48 \\
GBM & Macrophage & 0.03 & 0.58 \\
\hline
\end{tabular}

AEBP1, adipocyte enhancer binding protein 1; GBM, glioblastoma.

Table 2 Univariate analysis of the correlation between immune infiltration and AEBP1 expression with OS in GBM patients

\begin{tabular}{lcc}
\hline Cancer & Variable & $P$ \\
\hline GBM & Dendritic cell & 0.00 \\
GBM & AEBP1 & 0.00 \\
GBM & CD4 T cell & 0.02 \\
GBM & B Cell & 0.11 \\
GBM & Neutrophil & 0.74 \\
GBM & Macrophage & 0.77 \\
GBM & CD8 + cell & 0.81 \\
\hline
\end{tabular}

AEBP1, adipocyte enhancer binding protein 1; OS, overall survival; GBM, glioblastoma.

Table 3 Multivariate analysis of the correlation between immune infiltration and AEBP1 expression with OS in GBM patients

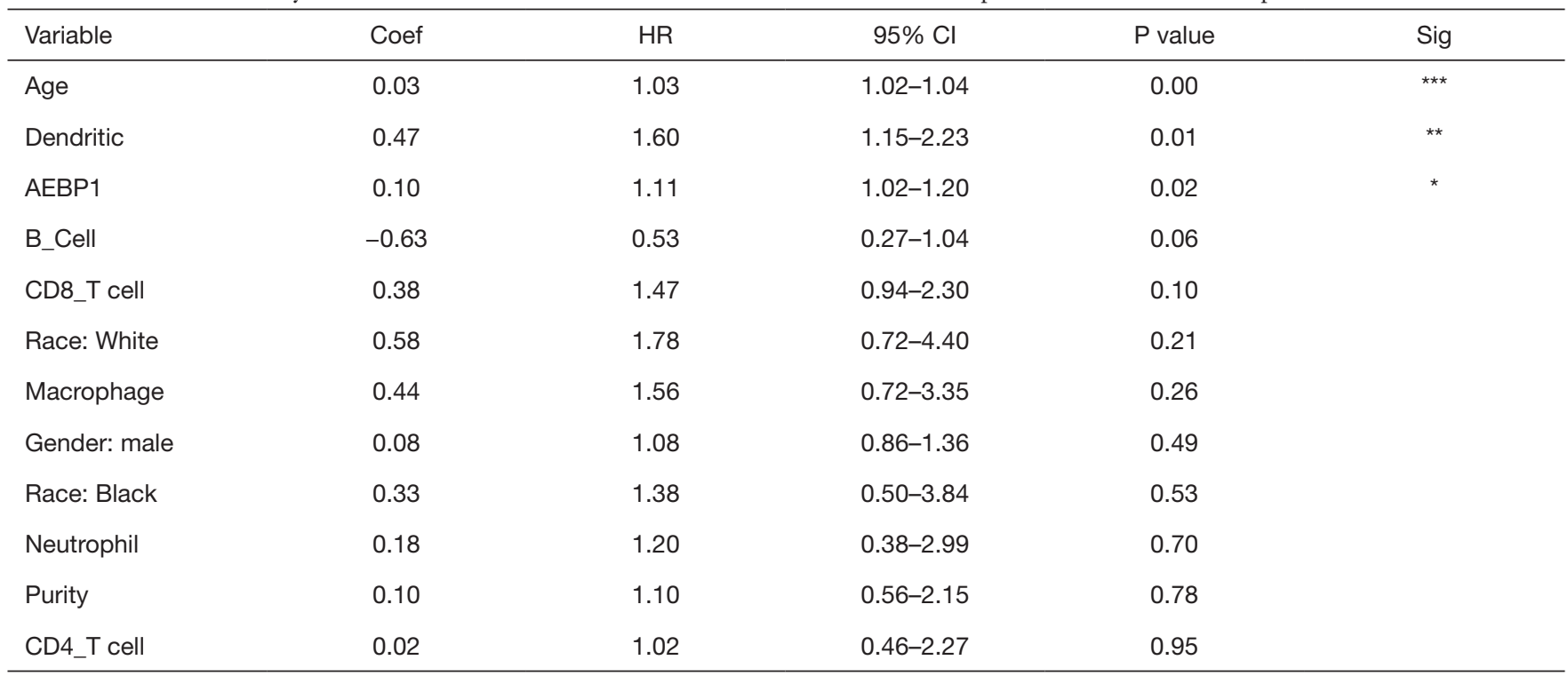

${ }^{*}, \mathrm{P}<0.05 ;{ }^{* *}, \mathrm{P}<0.01$; ${ }^{* \star}, \mathrm{P}<0.001$. AEBP1, adipocyte enhancer binding protein 1; OS, overall survival; GBM, glioblastoma. 
that AEBP1 appears to play a crucial regulatory role in the initiation and progression of GBM (40). We also found that the AEBP1 expression pattern was positively related with survival outcomes, which indicates that AEBP1 might be an effective marker in predicting the prognosis of GBM patients. In a further analysis, using data from the CGGA database, we once again confirmed the predictive role of AEBP1 in GBM.

Chemotherapy and radiotherapy are classic treatments for GBM. However, in our clinical investigation, we demonstrated the therapeutic superiority of chemotherapy to radiotherapy. Consistent with the conclusions of previous research, we also found that IDH mutation is a key factor affecting prognosis, and the expression of AEBP1 in IDH wild-type GBM is also significantly different from that in IDH mutant GBM cases. Thus, AEBP1 might be a key driver oncogene that promotes the tumorigenesis of GBM.

In relation to our immune analyses, the KEGG and GSEA results showed that immunity-related signaling pathways were significantly involved in the regulation network of AEBP1 (41). Molecularly, the TIMER analysis highlighted the inducible role of copy number alteration in the process of AEBP1 increasing immune infiltration. Specifically, dendritic cell infiltration might increase the negative risk of GBM (42). It has been reported that AEBP1 may promote the proliferation and progression of glioma cells in GBM through the NF- $\mathrm{KB}$ pathway and its downstream targets, such as MMP-2 and Bcl-2 (43). NF$\kappa \mathrm{B}$ is associated with immunity in tumors $(44,45)$, and $\mathrm{NF}-\kappa \mathrm{B}$ activity promotes tumor growth by inhibiting the infiltration, proliferation and immune response of effector $\mathrm{T}$ cells (45). we may begin to study the NF- $\mathrm{kB}$ pathway and immune infiltration in GBM in future studies. This novel finding might be used to develop more promising immunotherapies for GBM (46).

In conclusion, the survival rate of GBM patients decreased as AEBP1 expression levels increased. Further, Chemotherapy had an encouraging therapeutic effect on GBM. Additionally, the infiltration of immune cells might be regulated by the expression pattern of AEBP1. The immune-related pathways were also abundantly enriched in AEBP1. These novel findings are expected to lead to the development of novel solutions for GBM treatment. However, it should be noted that only public databases (i.e., TCGA and the CGGA) were employed to analyze and confirm the relationship between AEBP1 expression and prognosis using bioinformatic methods, and our study lacks more in vivo and in vitro evidence. After we establish an appropriate design and make the appropriate preparations, we will conduct further research on the molecular functions and mechanisms of AEBP1 in GBM through a series of cell-line, tissue, and animal experiments.

\section{Acknowledgments}

Funding: This work was supported by funding from the National Natural Science Foundation of China (NSFC) [81471178 (G-YY); 81522015; 81371305 (YW)] and the Science and Technology Commission of Shanghai Municipality \#13ZR1422600 (ZZ) and the SJTU National Infrastructures for Translational Medicine (Shanghai) Research Fund (No. TMSK-2020-112).

\section{Footnote}

Reporting Checklist: The authors have completed the REMARK reporting checklist. Available at https://dx.doi. org/10.21037/atm-21-5183

Conflicts of Interest: All authors have completed the ICMJE uniform disclosure form (available at https://dx.doi. org/10.21037/atm-21-5183). The authors have no conflicts of interest to declare.

Ethical Statement: The authors are accountable for all aspects of the work in ensuring that questions related to the accuracy or integrity of any part of the work are appropriately investigated and resolved. The study was conducted in accordance with the Declaration of Helsinki (as revised in 2013).

Open Access Statement: This is an Open Access article distributed in accordance with the Creative Commons Attribution-NonCommercial-NoDerivs 4.0 International License (CC BY-NC-ND 4.0), which permits the noncommercial replication and distribution of the article with the strict proviso that no changes or edits are made and the original work is properly cited (including links to both the formal publication through the relevant DOI and the license). See: https://creativecommons.org/licenses/by-nc-nd/4.0/.

\section{References}

1. Chen R, Smith-Cohn M, Cohen AL, et al. Glioma Subclassifications and Their Clinical Significance. Neurotherapeutics 2017;14:284-97. 


\section{Page 16 of 17}

2. Malta TM, de Souza CF, Sabedot TS, et al. Glioma CpG island methylator phenotype (G-CIMP): biological and clinical implications. Neuro Oncol 2018;20:608-20.

3. Peters KB. Revisiting anti-angiogenic therapy for recurrent glioblastoma. Transl Cancer Res 2019;8:S569-72.

4. Park CK, Jung JH, Park SH, et al. Multifarious proteomic signatures and regional heterogeneity in glioblastomas. J Neurooncol 2009;94:31-9.

5. Somasundaram K, Reddy SP, Vinnakota K, et al. Upregulation of ASCL1 and inhibition of Notch signaling pathway characterize progressive astrocytoma. Oncogene 2005;24:7073-83.

6. Yin J, Liu JS, Feng M, et al. Comprehensively investigating the expression levels and the prognostic role of transforming growth factor beta-induced (TGFBI) in glioblastoma mul-tiforme. Transl Cancer Res 2020;9:6487-504.

7. Kroes RA, Dawson G, Moskal JR. Focused microarray analysis of glyco-gene expression in human glioblastomas. J Neurochem 2007;103 Suppl 1:14-24.

8. He GP, Muise A, Li AW, et al. A eukaryotic transcriptional repressor with carboxypeptidase activity. Nature 1995;378:92-6.

9. Li S, Liu X, Liu T, et al. Identification of Biomarkers Correlated with the TNM Staging and Overall Survival of Patients with Bladder Cancer. Front Physiol 2017;8:947.

10. Cheon DJ, Tong Y, Sim MS, et al. A collagen-remodeling gene signature regulated by TGF- $\beta$ signaling is associated with metastasis and poor survival in serous ovarian cancer. Clin Cancer Res 2014;20:711-23.

11. Ladha J, Sinha S, Bhat V, et al. Identification of genomic targets of transcription factor AEBP1 and its role in survival of glioma cells. Mol Cancer Res 2012;10:1039-51.

12. Cheng L, Shao X, Wang Q, et al. Adipocyte enhancer binding protein 1 (AEBP1) knockdown suppresses human glioma cell proliferation, invasion and induces early apoptosis. Pathol Res Pract 2020;216:152790.

13. Wang K, Huang R, Tong X, et al. Molecular Characterization of AEBP1 at Transcriptional Level in Glioma. Biomed Res Int 2021;2021:5579359.

14. Jackson CM, Choi J, Lim M. Mechanisms of immunotherapy resistance: lessons from glioblastoma. Nat Immunol 2019;20:1100-9.

15. Gene Ontology C. The Gene Ontology (GO) project in 2006. Nucleic Acids Res 2006;34:D322-6.

16. Rickman DS, Bobek MP, Misek DE, et al. Distinctive molecular profiles of high-grade and low-grade gliomas

\section{Liu et al. AEBP1 is associated with the prognosis of glioblastoma}

based on oligonucleotide microarray analysis. Cancer Res 2001;61:6885-91.

17. Liang Y, Diehn M, Watson N, et al. Gene expression profiling reveals molecularly and clinically distinct subtypes of glioblastoma multiforme. Proc Natl Acad Sci U S A 2005;102:5814-9.

18. Lee J, Kotliarova S, Kotliarov Y, et al. Tumor stem cells derived from glioblastomas cultured in bFGF and EGF more closely mirror the phenotype and genotype of primary tumors than do serum-cultured cell lines. Cancer Cell 2006;9:391-403.

19. Sun L, Hui AM, Su Q, et al. Neuronal and glioma-derived stem cell factor induces angiogenesis within the brain. Cancer Cell 2006;9:287-300.

20. Bredel M, Bredel C, Juric D, et al. Functional network analysis reveals extended gliomagenesis pathway maps and three novel MYC-interacting genes in human gliomas. Cancer Res 2005;65:8679-89.

21. Tang Z, Li C, Kang B, et al. GEPIA: a web server for cancer and normal gene expression profiling and interactive analyses. Nucleic Acids Res 2017;45:W98-W102.

22. Goldman M, Craft B, Hastie M et al. The UCSC Xena platform for public and private cancer genomics data visualization and interpretation. bioRxiv. 2019.

23. Stajich JE, Block D, Boulez K, et al. The Bioperl toolkit: Perl modules for the life sciences. Genome Res 2002;12:1611-8.

24. Maag JLV. gganatogram: An R package for modular visualisation of anatograms and tissues based on ggplot2. F1000Res 2018;7:1576.

25. Dabbish LA, Stuart HC, Tsay J, Herbsleb JD. Social Coding in GitHub: Transparency and Collaboration in an Open Software Repository. CSCW '12: Proceedings of the ACM 2012 conference on Computer Supported Cooperative Work. Seattle, WA, USA. February 2012; 1277-1286.

26. Ritchie $\mathrm{ME}$, Phipson $\mathrm{B}, \mathrm{Wu} \mathrm{D}$, et al. limma powers differential expression analyses for RNA-sequencing and microarray studies. Nucleic Acids Res 2015;43:e47.

27. Kolde R. pheatmap: Pretty Heatmaps. 2015.

28. Yu G, Wang LG, Han Y, et al. clusterProfiler: an R package for comparing biological themes among gene clusters. OMICS 2012;16:284-7.

29. Walter W, Sánchez-Cabo F, Ricote M. GOplot: an R package for visually combining expression data with functional analysis. Bioinformatics 2015;31:2912-4.

30. Subramanian A, Tamayo P, Mootha VK, et al. Gene set enrichment analysis: a knowledge-based approach for 
interpreting genome-wide expression profiles. Proc Natl Acad Sci U S A 2005;102:15545-50.

31. Li T, Fan J, Wang B, et al. TIMER: A Web Server for Comprehensive Analysis of Tumor-Infiltrating Immune Cells. Cancer Res 2017;77:e108-10.

32. Mamelak AN, Jacoby DB. Targeted delivery of antitumoral therapy to glioma and other malignancies with synthetic chlorotoxin (TM-601). Expert Opin Drug Deliv 2007;4:175-86.

33. Chai R, Zhang K, Wang K, et al. A novel gene signature based on five glioblastoma stem-like cell relevant genes predicts the survival of primary glioblastoma. J Cancer Res Clin Oncol 2018;144:439-47.

34. Jiang T, Mao Y, Ma W, et al. CGCG clinical practice guidelines for the management of adult diffuse gliomas. Cancer Lett 2016;375:263-73.

35. Chai RC, Zhang KN, Liu YQ, et al. Combinations of four or more CpGs methylation present equivalent predictive value for MGMT expression and temozolomide therapeutic prognosis in gliomas. CNS Neurosci Ther 2019;25:314-22.

36. Atkins RJ, Ng W, Stylli SS, et al. Repair mechanisms help glioblastoma resist treatment. J Clin Neurosci 2015;22:14-20.

37. Stupp R, Mason WP, van den Bent MJ, et al. Radiotherapy plus concomitant and adjuvant temozolomide for glioblastoma. N Engl J Med 2005;352:987-96.

38. $\mathrm{Hu} \mathrm{W}$, Jin $\mathrm{L}$, Jiang CC, et al. AEBP1 upregulation confers acquired resistance to BRAF (V600E) inhibition in melanoma. Cell Death Dis 2013;4:e914.

Cite this article as: Liu M, Yu Y, Zhang Z, Chen Z, Chen B, Cheng Y, Wei Y, Li J, Shang H. AEBP1 as a potential immunerelated prognostic biomarker in glioblastoma: a bioinformatic analyses. Ann Transl Med 2021;9(22):1657. doi: 10.21037/atm21-5183
39. Liu JY, Jiang L, Liu JJ, et al. AEBP1 promotes epithelialmesenchymal transition of gastric cancer cells by activating the NF- $\kappa \mathrm{B}$ pathway and predicts poor outcome of the patients. Sci Rep 2018;8:11955.

40. Sinha S, Renganathan A, Nagendra PB, et al. AEBP1 down regulation induced cell death pathway depends on PTEN status of glioma cells. Sci Rep 2019;9:14577.

41. O'Donnell JS, Madore J, Li XY, et al. Tumor intrinsic and extrinsic immune functions of CD155. Semin Cancer Biol 2020;65:189-96.

42. Sokratous G, Polyzoidis S, Ashkan K. Immune infiltration of tumor microenvironment following immunotherapy for glioblastoma multiforme. Hum Vaccin Immunother 2017;13:2575-82.

43. Majdalawieh AF, Massri M, Ro HS. AEBP1 is a Novel Oncogene: Mechanisms of Action and Signaling Pathways. J Oncol 2020;2020:8097872.

44. Takenaka MC, Gabriely G, Rothhammer V, et al. Control of tumor-associated macrophages and $\mathrm{T}$ cells in glioblastoma via AHR and CD39. Nat Neurosci 2019;22:729-40.

45. Dumas AA, Pomella N, Rosser G, et al. Microglia promote glioblastoma via mTOR-mediated immunosuppression of the tumour microenvironment. EMBO J 2020;39:e103790.

46. Kwok D, Okada H. T-Cell based therapies for overcoming neuroanatomical and immunosuppressive challenges within the glioma microenvironment. J Neurooncol 2020;147:281-95.

(English Language Editor: L. Huleatt) 